\title{
Dynamical-Decoupling-Based Quantum Sensing: Floquet Spectroscopy
}

\author{
J. E. Lang, ${ }^{1}$ R. B. Liu, ${ }^{2}$ and T. S. Monteiro ${ }^{1, *}$ \\ ${ }^{1}$ Department of Physics and Astronomy, University College London, \\ Gower Street, London WC1E 6BT, United Kingdom \\ ${ }^{2}$ Department of Physics, The Chinese University of Hong Kong, Hong Kong, China \\ (Received 5 June 2015; revised manuscript received 7 September 2015; published 30 October 2015)

\begin{abstract}
Sensing the internal dynamics of individual nuclear spins or clusters of nuclear spins has recently become possible by observing the coherence decay of a nearby electronic spin: the weak magnetic noise is amplified by a periodic, multipulse decoupling sequence. However, it remains challenging to robustly infer underlying atomic-scale structure from decoherence traces in all but the simplest cases. We introduce Floquet spectroscopy as a versatile paradigm for analysis of these experiments and argue that it offers a number of general advantages. In particular, this technique generalizes to more complex situations, offering physical insight in regimes of many-body dynamics, strong coupling, and pulses of finite duration. As there is no requirement for resonant driving, the proposed spectroscopic approach permits physical interpretation of striking, but overlooked, coherence decay features in terms of the form of the avoided crossings of the underlying quasienergy eigenspectrum. This is exemplified by a set of "diamond-shaped" features arising for transverse-field scans in the case of single-spin sensing by nitrogen-vacancy centers in diamond. We also investigate applications for donors in silicon, showing that the resulting tunable interaction strengths offer highly promising future sensors.
\end{abstract}

DOI: $10.1103 / P h y s R e v X .5 .041016$

Subject Areas: Atomic and Molecular Physics, Nanophysics, Quantum Physics

\section{INTRODUCTION}

There is enormous interest in the rapidly advancing field of detection and imaging at the single-spin level [1-4], mainly with nitrogen-vacancy (NV) color centers but also other defects in diamond [5,6], not only as a source of versatile qubits for quantum information [7-10] and entanglement generation [11], but principally because they underpin a new generation of quantum sensors, for magnetometry and atomic-scale characterization of the environment [12-18]. In the widely studied case of dynamicaldecoupling quantum sensing, a sequence of pulses modulates the coherent evolution of the sensor and, in some cases, sharp "dips" in coherence allow one to detect, and infer useful characteristics of, nearby single spins or small spin clusters. Complexities in the environment being studied mean that the single isolated sharp dip is found in a restricted subset of the data and motivates the development of more general or alternative methods of analysis. In particular, many decoupling sequences are temporally periodic.

In this case, Floquet's theorem provides a canonical form for the solution of periodically driven systems and has

\footnotetext{
* Corresponding author. t.monteiro@ucl.ac.uk

Published by the American Physical Society under the terms of the Creative Commons Attribution 3.0 License. Further distribution of this work must maintain attribution to the author $(s)$ and the published article's title, journal citation, and DOI.
}

found wide applicability in various branches of quantum physics since 1965 [19], especially in light-matter interactions with continuous driving and multiphoton atomic physics. Floquet's theorem can be applied to any periodic quantum Hamiltonian for which $\hat{H}\left(t+\tau_{\text {tot }}\right)=\hat{H}(t)$. In practical implementations, instead of analyzing the eigenstates of the static Hamiltonian, which are appropriate only in the perturbative limit of weak driving, one employs instead the eigenspectrum of the one-period time-evolution propagator. Floquet theory is also employed in the analysis of nuclear magnetic resonance (NMR) spectra and related fields [20], where applications are essentially limited to resonant and continuous driving.

But this approach has not been considered for analysis of coherence behaviors in this new generation of multipulse spin sensing experiments. We argue that the Floquet spectroscopy method proposed is better adapted to regimes of strong quantum entanglement between the sensor and detected spin systems than signal processing methods applied to classical ac signals, or geometric approaches based on two-state systems. In this work, we find that Floquet theory can augment current methods of analysis in several ways:

(1) Floquet theory is equally applicable to off resonant as to resonant driving. The understanding of spinsensing data is often cast in signal processing terms: the multipulse sequence imposes a filter function which selects an ac signal with a reasonably well-defined characteristic frequency $\omega_{a c} / 2 \pi$, which may, in turn, be used to infer interatomic coupling 

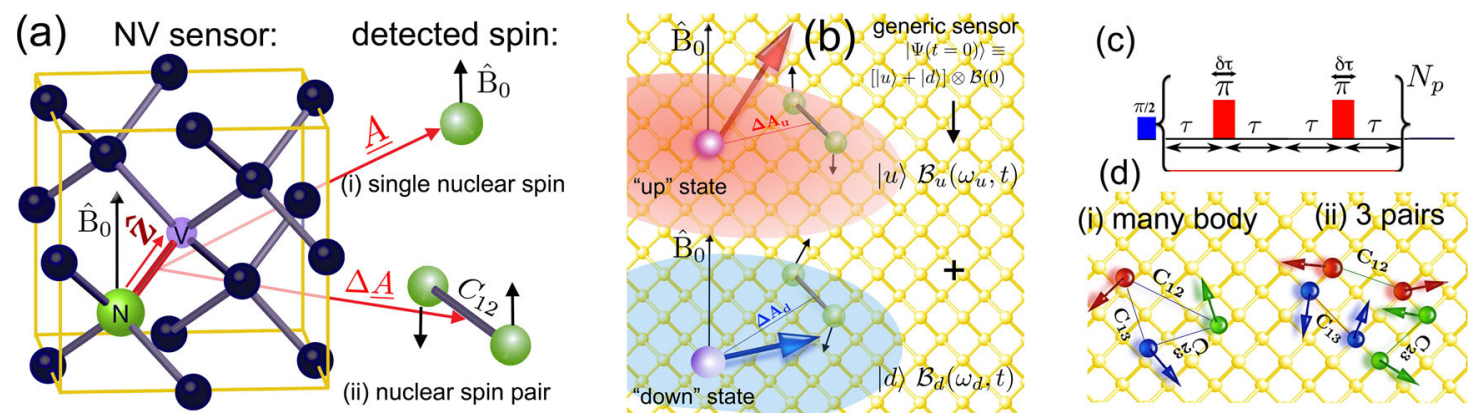

FIG. 1. (a) Current experiments have employed the $S=1$ electronic spins of NV centers to successfully detect (i) single nuclear spins [15,21,22], (ii) the internal dynamics of nuclear spin pairs [16], as well to characterize on the atomic scale, by estimating parameters such as electronic-nuclear dipolar couplings $A$ and internuclear dipolar couplings $C_{12}$. (b) Generic sensor detecting a pair of nuclear spins. The electronic spin state is in a superposition of "up" $|u\rangle$ and "down" $|d\rangle$ states. The nuclear dynamics and its characteristic frequencies $\omega_{u, d}$ depend on the electronic state. In turn, the electronic coherence is sensitive to the resulting weak ac noise from the nuclei. This may be amplified by dynamical decoupling control such as CPMG, leading to observed "dips" in coherence. These are at well-defined frequencies in typical weak-coupling regimes where the nuclear dynamics is not too different in the $u, d$ subspaces. However, strong-coupling regimes do not necessarily yield sharp dips. (c) Additional complexities occur for pulses of finite durations. (d) It is also challenging to differentiate between (i) independent pairs of spins and (ii) many-body effects from an equivalent interacting cluster. Floquet theory is not restricted to single spins or spin pairs and can be applied also to analysis of larger, correlated spin clusters, strong coupling, and off-resonant driving.

parameters. When $\omega_{\mathrm{ac}}$ is resonant with the pulse interval $\tau$ [see Fig. 1(c)], so when $\omega_{\mathrm{ac}} / \pi=1 /(4 \tau)$, a narrow dip in coherence is observed. Here, we show that away from such resonances, or even for broadened resonances, Floquet theory can shed insight on other striking features that are not narrow dips but can, nevertheless, yield rich information for atomicscale characterization. The key reason is that we show that the widths and shapes of these features may be understood in terms of avoided crossings of an underlying Floquet spectrum.

(2) Current experiments operate primarily in regimes of weak coupling. The pulses involve consecutive switching between two electronic states $u, d$; the associated characteristic frequencies of the detected spins $\omega_{u}, \omega_{d}$ can vary significantly, since, for stronger coupling, there is significant backaction and entanglement between the sensor and detected spins. For weak coupling, $\omega_{u} \simeq \omega_{d}$, and, in addition, average Hamiltonian theory models apply, predicting typically $\omega_{\text {ac }} \approx \frac{1}{2}\left(\omega_{u}+\omega_{d}\right)$. Floquet theory remains valid regardless of coupling strength; we examine regimes of failure, obtain alternative forms for $\omega_{\mathrm{ac}}$, and show that the avoided crossings shed insight into these regimes. It also remains valid even if there is nontrivial evolution due to finite duration of the pulses, a problem only recently identified [17].

(3) For detection of two-state systems (spins or spin pairs that reduce to an effective pseudospin), geometric methods [16,21] are widely used to interpret data and yield analytical expressions for the coherence decays. Although here the Floquet method already sheds additional physical insight, its full value is that it is universally valid even for higher-dimensional state spaces, and so would facilitate studies of, e.g., multispin clusters.

The key features of dynamical-decoupling-based quantum sensing, using a multipulse periodic sequence, are illustrated in Fig. 1. A $\pi / 2$ pulse prepares the sensor system in a superposition state, $\psi(t=0)=(1 / \sqrt{2})(|u\rangle+$ $|d\rangle) \otimes \mathcal{B}(0)$, where $\mathcal{B}(0)$ is the detected spin cluster at initial time. In turn, interaction with the sensor means that the spin cluster becomes entangled with the sensor, $\psi(t) \simeq(1 / \sqrt{2})\left[|u\rangle \mathcal{B}\left(\omega_{u}, t\right)+|d\rangle \mathcal{B}\left(\omega_{d}, t\right)\right]$, where $\mathcal{B}\left(\omega_{u, d}, t\right)=$ $\left(\hat{T}_{u, d}\right)^{N_{p}} \mathcal{B}(0)$, for a pulse sequence (with propagator $\hat{T}_{u, d}$ ), which is repeated $N_{p}$ times. The detected spin dynamics is associated with a characteristic frequency that is state dependent. The temporal coherence $\mathcal{L}(t)=\left\langle S^{+}\right\rangle$is given by $\mathcal{L}(t)=\left\langle\mathcal{B}\left(\omega_{u}, t\right) \mid \mathcal{B}\left(\omega_{d}, t\right)\right\rangle$ to within a normalization factor; averaged over bath states, it simulates the experimentally measured signal.

\section{FLOQUET THEORY}

Floquet's theorem is generally applicable to periodically forced systems, classical or quantum, but it allows one specifically to write solutions to the Schrödinger equation in terms of quasienergy states, $\left|\psi_{l}(t)\right\rangle=\exp \left(-i \epsilon_{l} t\right)\left|\Phi_{l}\right\rangle$, where $\epsilon_{l}$ is the quasienergy, $\left|\Phi_{l}(t)\right\rangle=\left|\Phi_{l}\left(t+\tau_{\text {tot }}\right)\right\rangle, \tau_{\text {tot }}$ is the period, and $l=1, \ldots, D$ ( $D$ is the dimension). However, for problems (such as our present study) where we require only "stroboscopic" knowledge of our system (i.e., read-out once every period $\tau_{\text {tot }}$ ), the solution is even simpler. We can obtain Floquet phases or modes simply as the eigenvalues or eigenstates of the one-period unitary evolution operator $\hat{T}\left(\tau_{\text {tot }}, 0\right)$. The Floquet modes $\left|\Phi_{l}\right\rangle$ obey the eigenvalue equation: 


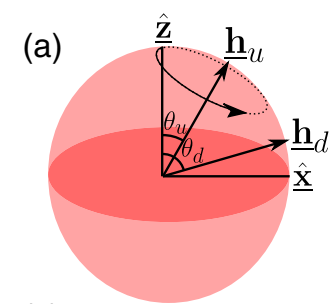

(b)

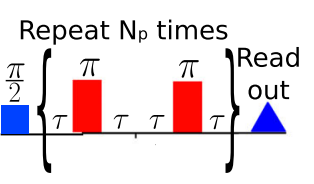

(c)

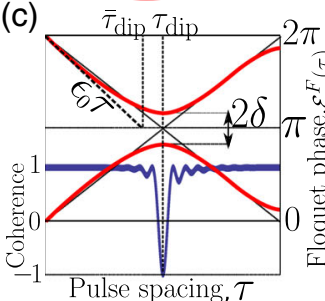

(d)

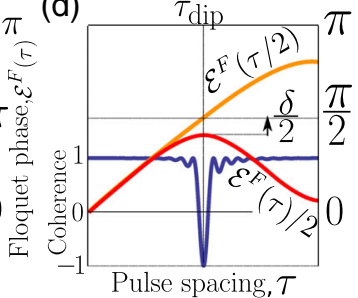

(e)

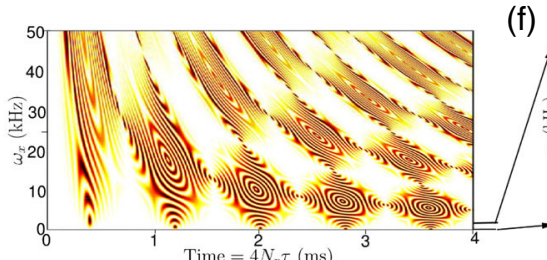

(f)

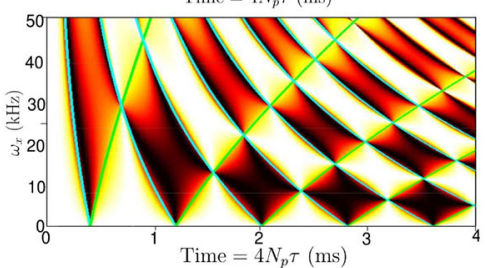

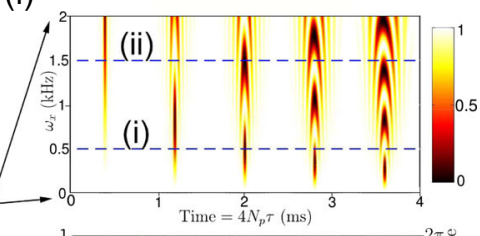
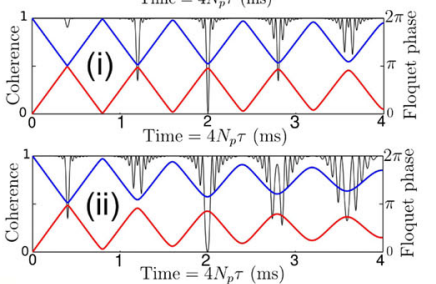

FIG. 2. (a) Usual geometric approach. Under CPMG-N control (b) the detected spins represent two-state systems that precess about effective magnetic field, depending on the "up" $|u\rangle$ and "down" $|d\rangle$ states of the probe spin. The coherence dips are understood by following the precessions and relative angles between these spins, with increasing $N$. (c) Spectroscopic picture. The dips in coherence occur at avoided crossings of the Floquet eigenstates. Both the position and contrast of the decoherence dip are related to the curvature of the crossing. This is characterized by the splitting between the states $2 \delta$ and the deviation from the early $\tau \rightarrow 0$ linear evolution. The early time evolution (the $\epsilon_{0}$ quasienergy) gives the dip position $\bar{\tau}_{\text {dip }}$ for average Hamiltonian theory; the coherence minimum is given by $\mathcal{E}\left(\tau_{\text {dip }} / 2\right)=\pi / 2$. (d) The dip contrast depends on the degree of curvature of the crossing, characterized by the level-repulsion strength, $\delta=2\left[\mathcal{E}\left(\tau_{\text {dip }}\right) / 2-\mathcal{E}\left(\tau_{\text {dip }} / 2\right)\right]$. (e) NV-center decoherence "diamonds." While typical experimental studies scan along parallel field $\left(\omega_{z}\right)$ component (thus remaining in weak-coupling, single-dip regime), scanning the transverse magnetic field $\left(\omega_{x}\right)$ would produce diamond pattern of high-decoherence regions, as avoided crossings widen (and even overlap) then narrow (here, $\omega_{z}=0$ and $A_{\|}=50 \mathrm{kHz}$ ). The upper panel shows full oscillating coherence function, for $N_{p}=10$ pulse pairs; the lower panel shows coherence envelopes, filled as $N_{p} \rightarrow \infty$. Here, $\omega_{z}=0$. Boundaries of the diamonds trace out (green) $\tau=\pi / 2\left(\omega_{d}+\omega_{u}\right)$ and (cyan) $\tau=\pi / 2\left(\omega_{d}-\omega_{u}\right)$ (see text). (f) Expanded version of low-field region showing shape of avoided crossings versus coherence traces corresponding to two cuts (i),(ii), indicated in the upper panel.

$$
\hat{T}\left(\tau_{\text {tot }}\right)\left|\Phi_{l}\right\rangle=\lambda_{l}\left|\Phi_{l}\right\rangle \equiv \exp \left(-i \mathcal{E}_{l}\right)\left|\Phi_{l}\right\rangle,
$$

where now $\mathcal{E}_{l}$ is the eigenphase (the Floquet phase) and $\epsilon_{l}=$ $\mathcal{E}_{l} / \tau_{\text {tot }}$ is the quasienergy. For sensing, we can obtain Floquet phases or modes simply as the eigenvalues or eigenstates of $\hat{T}_{u, d}$, the basic periodic sequence; for example, for the CarrPurcell-Meiboom-Gill (CPMG) sequence in Fig. $2, \tau_{\text {tot }}=4 \tau$. In that instance $\hat{T}_{i}\left|\Phi_{i l}\right\rangle=e^{-i \mathcal{E}_{l}^{(i)}}\left|\Phi_{i l}\right\rangle$, where $i=u, d$ denotes the state of the sensor spin. The eigenphases for $N_{p}=1$ fully determine the time evolution of the system since if the pulse sequence is repeated $N_{p}$ times, we just scale the eigenphases, so for longer time propagation,

$$
\left(\hat{T}_{i}\right)^{N_{p}}\left|\Phi_{i l}\right\rangle=e^{-i N_{p} \mathcal{E}_{l}^{(i)}}\left|\Phi_{i l}\right\rangle .
$$

In the present work, we show that these Floquet eigenphases and eigenstates have particular important properties.

(a) The eigenvalues are the same for the upper and lower states; i.e., $e^{i \mathcal{E}_{l}^{(u)}}=e^{i \mathcal{E}_{l}^{(d)}} \equiv e^{i \mathcal{E}_{l}}$. This holds even for pulses of finite duration, in typical cases. In other words, the evolution of the $\hat{T}_{u}^{\left(N_{p}\right)} \mathcal{B}(0)$ and $\hat{T}_{d}^{\left(N_{p}\right)} \mathcal{B}(0)$ are characterized by the same set of effective frequencies $\epsilon_{l}=\mathcal{E}_{l} / \tau_{\text {tot }}$, in contrast to typical static, geometric approaches where two distinct sets of frequencies $\omega_{u l}$ and $\omega_{d l}$ are involved.

(b) The eigenvectors do not, in general, coincide, but we show that (to within a phase term) the eigenvectors are related to each other by a half-period evolution; e.g., $\hat{T}_{u}\left(\tau_{\text {tot }} / 2\right)\left|\Phi_{u l}\right\rangle \propto\left|\Phi_{d l}\right\rangle$.

(c) Minima in coherence (of prime importance for sensing, whether sharp dips or not) occur at avoided crossings of Floquet eigenstates, where $e^{i \mathcal{E}_{l}} \simeq e^{i \mathcal{E}_{k}}$. Once the Floquet phases and modes are obtained, one can obtain the general form of the decoherence for arbitrary times, which, averaged over bath states, yields

$$
\left\langle\mathcal{L}\left(t=N_{p} 4 \tau\right)\right\rangle=\frac{1}{D} \sum_{l, l^{\prime}}^{D} e^{-i N_{p}\left(\mathcal{E}_{l}-\mathcal{E}_{l^{\prime}}\right)}\left|\left\langle\Phi_{d l^{\prime}} \mid \Phi_{u l}\right\rangle\right|^{2} .
$$

Derivations of (a)-(c) are given in Appendix A-C. Although properties (a)-(c) are quite generic, physical insight on the Floquet picture is more easily gained from two-state systems, where direct comparison with usual geometric methods $[21,23,24]$ is also possible. For the twostate case, eigenvalues must be conjugate pairs, $\lambda_{ \pm}=e^{ \pm i \mathcal{E}}$. Level crossings occur when $\lambda_{+} \simeq \lambda_{-}$; hence, the crossings must occur at $\mathcal{E} \simeq 0, \pi, 2 \pi$. The generic properties of states 
at avoided crossings (see Appendix A-C) then imply that coherence dips occur at $\mathcal{E} \simeq \pi$. We now first investigate the Floquet dynamics for these two-state single-spin or singlepseudospin models.

\section{A. Single-spin or spin-pair detection}

Both pair flip-flop dynamics as well as single-spin dynamics (in systems like NV centers where a crystal field leads to nontrivial one-spin dynamics) can be approximated by a two-state Hamiltonian. We term this the pseudospin model, noting that for single-spin detection there is a genuine spin, while for pair dynamics $[14,16]$ it is a pseudospin. It has led to a successful, widely used geometric model [see Fig. 2(a) and Ref. [25]], where the evolution of the pseudospin is conditional on the state $i=u, d$ of the probe and corresponds to precession about an effective magnetic field: $H_{i}=\frac{1}{2} \mathbf{h}^{i} \cdot \boldsymbol{\sigma}=\frac{1}{2}\left(X \sigma_{x}+Z_{i} \sigma_{z}\right)$, where $\sigma_{x}, \sigma_{z}$ are Pauli matrices in the usual spin basis; in the pseudospin case, of course, we have $|\uparrow \downarrow\rangle \rightarrow|\uparrow\rangle$ and $|\downarrow \uparrow\rangle \rightarrow|\downarrow\rangle)$ ). The $X, Z_{i}$ depend on the physical system (see Ref. [25] for details), but for NV centers $\mathbf{h}^{u} \simeq\left(\omega_{x}, 0, A_{\|}+\right.$ $\left.\omega_{z}\right)$, while $\mathbf{h}^{d} \simeq\left(\omega_{x}, 0, \omega_{z}\right)$, where $\gamma \mathbf{B}_{0}=\left(\omega_{x}, 0, \omega_{z}\right)$ is the external magnetic field, $\gamma$ is the gyromagnetic ratio and $A_{\|}$ the parallel component of the hyperfine interaction. For spin-pair sensing, on the other hand, $\mathbf{h}^{i}=\frac{1}{2}\left(C_{12}, 0, \Delta A_{i}\right)$, where $\Delta A_{i}=2\left(A_{1}-A_{2}\right)\left\langle i\left|\hat{S}_{z}\right| i\right\rangle$ represents the energy detuning between the nuclear spins in the pair and $\hat{S}$ represents the operator for the sensor spin. The eigenvalues of $H_{i}$ are $\omega_{u, d}= \pm \frac{1}{2} \sqrt{X^{2}+Z_{u, d}^{2}}$ and the orientation of the effective field is $\theta_{i}=\arctan \left(X / Z_{i}\right)$. For two-state systems, we obtain

$$
\langle\mathcal{L}(\tau)\rangle=1-2\left[\frac{\cos ^{2}[\mathcal{E}(\tau) / 2]-\cos ^{2}[\mathcal{E}(\tau / 2)]}{\cos ^{2}[\mathcal{E}(\tau) / 2]}\right] \sin ^{2}\left[N_{p} \mathcal{E}(\tau)\right] .
$$

This is a key result of the present work as it means one can give the full coherence function using only the Floquet phases. To calculate the Floquet eigenphase $\mathcal{E}(\tau)$, as well as its halfperiod value $\mathcal{E}(\tau / 2)$ in Eq. (4), one may use $\cos [\mathcal{E}(s)]=$ $\cos \left(2 \omega_{u} s\right) \cos \left(2 \omega_{d} s\right)-\sin \left(2 \omega_{u} s\right) \sin \left(2 \omega_{d} s\right) \cos \left(\theta_{u}-\theta_{d}\right)$, with $s=\tau$ or $s=\tau / 2$. Thus, the coherence takes the form $\mathcal{L}(\tau)=1-F(\tau) \sin ^{2}\left[N_{p} \mathcal{E}(\tau)\right]$, which is the product of a smooth envelope $F(\tau)$, independent of $N_{p}$, superimposed on a fast oscillating function $\sin ^{2}\left[N_{p} \mathcal{E}(\tau)\right]$, dependent on $N_{p}$.

A full comparison with geometric methods is in Ref. [25] where we argue it is the condition

$$
\mathcal{E}\left(\tau_{\text {dip }} / 2\right)=\pi / 2
$$

that best specifies the dip positions. The depth of the dip is related to the eigenvalue splitting parameter $\delta=\pi-\mathcal{E}\left(\tau_{\text {dip }}\right)$, at the dip

$$
\mathcal{L}\left(t=4 N_{p} \tau_{\mathrm{dip}}\right)=1-2 \sin ^{2}\left(N_{p} \delta\right) .
$$

Hence, if $\mathcal{E}=\pi$, there is no dip, so a true level crossing provides no signal. For $N_{p} \delta \gtrsim \pi / 2$, the width and shape of the dip becomes largely independent of $N_{p}$ and is fully determined by the Floquet anticrossing and envelope function, since the $\sin ^{2}\left(N_{p} \delta\right)$ prefactor simply superposes fast oscillations on $F(\tau)$. A narrow avoided crossing (low splitting, $\delta$ small) gives a single, sharp (but weaker) coherence dip, while a large $\delta$ crossing has a broad envelope. It is only for low $N_{p} \delta \ll \pi / 2$ that the dip height is strongly dependent on $N_{p}$; here, the central height increases as $\left(N_{p} \delta\right)^{2}$.

Comparison with average Hamiltonian models (see Ref. [25] for details). - A frequently used approximation in spin sensing is the average Hamiltonian model whereby the eigenvalues $\omega_{\mathrm{av}}$ of $\frac{1}{2}\left(H_{u}+H_{d}\right)$, the time-averaged Hamiltonian, provide an estimate of the dip positions and $T_{\text {dip }}=\left(N_{p} \pi / \omega_{\mathrm{av}}\right)$. From Figs. 2(c) and 2(d), we can equate linear behavior in our eigenvalues (narrow crossing, linear shape) both with the occurrence of a sharp dip as well as validity of the averaged Hamiltonian model. In particular, for small $\tau, \mathcal{E}(\tau) \simeq 4 \epsilon_{0} \tau$ corresponds to the averaged Hamiltonian results. Expanding the $\cos \mathcal{E}(\tau)$ from below Eq. (4), for small $\tau$, we easily obtain $\epsilon_{0}=\frac{1}{2}\left[\omega_{u}^{2}+\omega_{d}^{2}+2 \omega_{u} \omega_{d} \cos \left(\theta_{u}-\theta_{d}\right)\right]^{1 / 2}$, and thus,

$$
\bar{\tau}_{\mathrm{dip}}=\frac{\pi}{2\left[\omega_{u}^{2}+\omega_{d}^{2}+2 \omega_{u} \omega_{d} \cos \left(\theta_{u}-\theta_{d}\right)\right]^{1 / 2}} .
$$

Expressing quantities in terms of the pseudofield components $X, Z_{u, d}$, we can show that this is equivalent to the expression $\omega_{\mathrm{av}}=\frac{1}{2}\left[\left(X^{2}+\left(Z_{u}+Z_{d}\right)^{2} / 4\right]^{1 / 2}\right.$ used in spindetection experiments $[14,16]$ and to $\omega_{\mathrm{av}} \simeq \frac{1}{2}\left(\omega_{u}+\omega_{d}\right)$ for $\theta_{u} \simeq \theta_{d}$.

\section{APPLICATIONS}

\section{A. Experimental control of quasienergy crossings}

The above motivates us to investigate possibilities for experimental control of the avoided crossings, by varying $\delta$, even in the simple one-spin or spin-pair case. In typical sensing with NV centers, we have $\omega_{z} \gtrsim \omega_{x} \gg A$, thus, in Eq. (7), we have $\theta_{u} \sim \theta_{d} \ll \pi$, thus, $\bar{\tau}_{\text {dip }} \simeq\left(\pi / 2\left(\omega_{u}+\omega_{d}\right)\right)$. However, setting $\omega_{z}=0$ and increasing $\omega_{x}$ causes the anticrossings to widen and narrow successively, forming a checkerboard pattern of diamonds. This behavior is illustrated in Figs. 2(e) and 2(f). In particular, Fig. 2(e) illustrates the usefulness of the 2D map; it is not easy to clearly discern the behavior from an individual trace [as in Fig. 2(f)], especially if $N_{p}$ is not very large. We note that higher harmonics have larger $\delta$ than lower harmonics at the same parameters. 
However, here we consider in addition $S=1 / 2$ systems as potential sensors. These might include silicon vacancies, but in particular we focus on electron donors in silicon. Although techniques analogous to optical read-out and polarization of NV centers are not fully developed, there has been considerable progress in single-spin detection [26-28]. These systems also benefit from extremely long coherence times (of order seconds) for cryogenically cooled samples. They are also an ideal test bed for the theory as one can vary $\theta_{u}, \theta_{d}$ over a wide range as magnetic field $B_{0}$ is swept. For donor systems, the surrounding ${ }^{29} \mathrm{Si}$ nuclear spin dynamics does not generate an ac signal as there is no internal crystal axis, in contrast to the case of NV centers in diamond where the surrounding nuclear spins precess around an effective quantization axis which is no longer only the external magnetic field. For donors, single
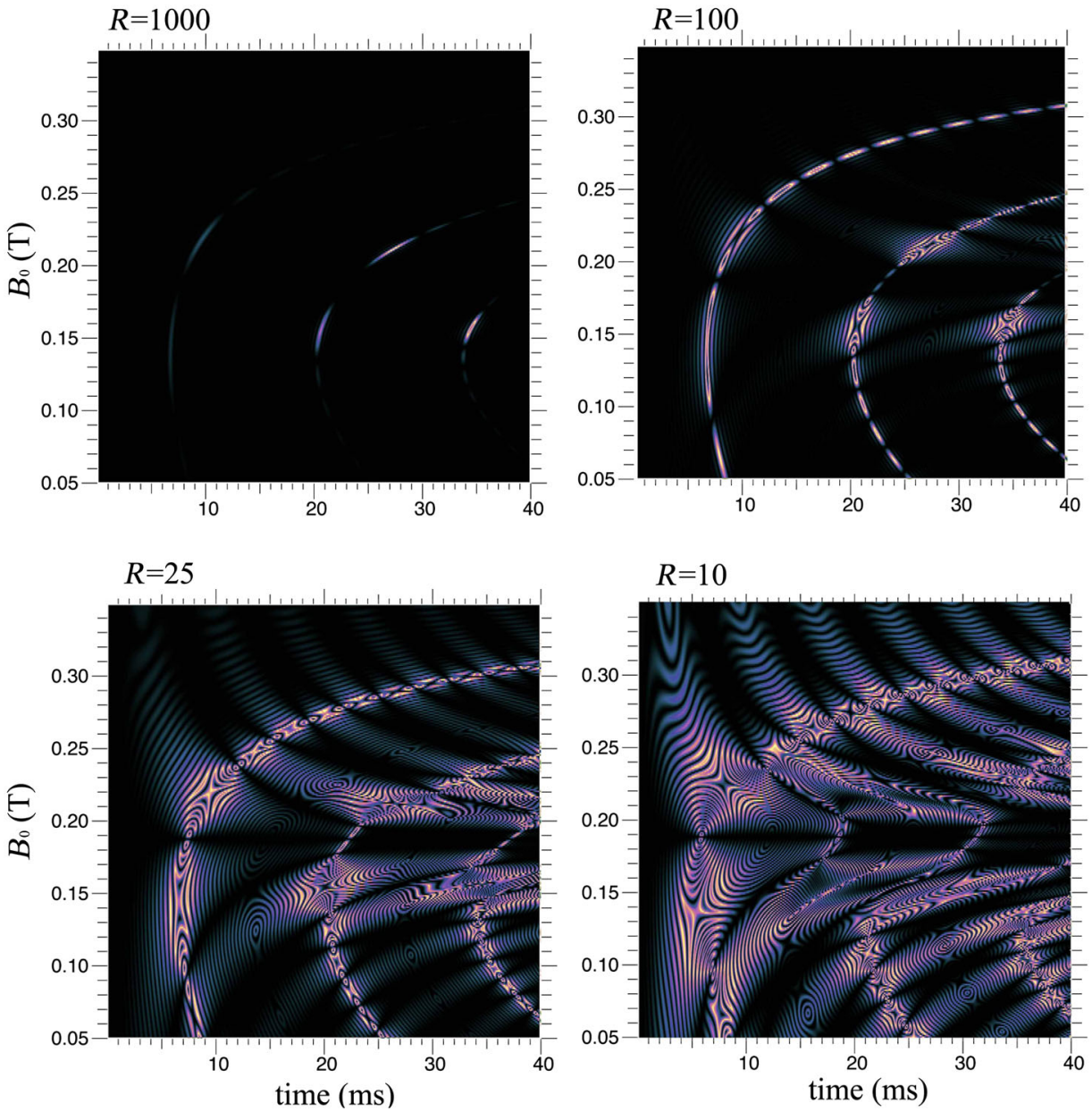

strongly coupled ${ }^{29} \mathrm{Si}$ nuclei have recently been detected via the static shift of the donor frequency [29]. However, the interesting coherence dynamics in these systems involve only pairs or larger clusters of spins where the flip-flopping dynamics generates an ac signal [30,31].

Formally, the state-conditional dynamics for donors is very similar to that for $\mathrm{NV}$ centers: The dynamics correspond to an effective spin precessing about effective magnetic fields, $\mathbf{h}^{i}=\frac{1}{2}\left(C_{12}, 0, \Delta A_{i}\right)$, where $\Delta A_{i}=\left(A_{1}-A_{2}\right) P_{i}$. But in contrast to NV centers $P_{i}\left(B_{0}\right)=2\left\langle i\left|\hat{S}_{z}\right| i\right\rangle$, the polarization of the state (see Ref. [25]) varies strongly with the magnetic field [32,33], while for NV centers, $\left\langle i\left|\hat{S}_{z}\right| i\right\rangle=0, \pm 1$ is fixed for the modest fields used in experiments.

Figure 3 shows the field dependence of the coherence for a variety of coupling strengths. The behavior may be compared with the NV centers: in this case, the coherence

FIG. 3. Coherence decay behavior for an electron spin detecting a flip-flopping pair of nuclear spins, for a donor in silicon system (see Ref. [25]) with tunable interactions. $\mathcal{L}\left(B_{0}, t\right)$ exhibits a rich structure in the two-dimensional $\tau, B_{0}$ plane, which is not evident in the normal traces at constant $B_{0}$. Decoherence map is shown for different $R=\Delta A / C_{12},\left(\Delta A=A_{1}-A_{2}\right)$. Large $R$ corresponds to weaker dipolar coupling $C_{12}$, and the maps trace the locus of a set of isolated sharp dips in coherence. For smaller $R$, there are no longer single dips; nevertheless, the envelopes [given by $F(\tau)$ ] are well defined and track the behavior of the underlying Floquet avoided crossings. The background oscillatory structure depends on $N_{p}$, the envelopes do not. Time $t \equiv 4 N_{p} \tau$ (color scale linear, where black is 1 , yellow is $<0.5$ ). Similar behavior is obtained for several transitions of Si:Bi and other donors, but specific parameters are for $12 \rightarrow 9$ ESR transition of $\mathrm{Si}: \mathrm{Bi}$ and $2 N_{p}=40$. 
dips trace out a curved locus. Like NV centers, for stronger $X \equiv C_{12} / 2$ component of the pseudofield, the envelopes broaden, but there is a similar pattern of intermittent broadening and narrowing. There is a striking feature at $B_{0}=188 \mathrm{mT}$ in Fig. 3 where all decoherence envelopes "collapse" to a sharp dip. This is one of a set of special fields [optimal working points (OWP)] where $\theta_{u} \simeq \theta_{d}$ and $\omega_{u} \simeq \omega_{d}$ and which have been investigated theoretically and experimentally for their favorable coherence properties $[31,32,34]$.

But, in the present work, we find that these points also correspond to very narrow Floquet avoided crossings, at which $\delta \rightarrow 0$. Figure 4 compares the dip position predicted by average Hamiltonian theory Eq. (7) with the accurate dip condition $\mathcal{E}\left(\tau_{\text {dip }} / 2\right)=\pi / 2$. In Fig. $4, \Delta A=\left(A_{1}-A_{2}\right)$ (which approximately sets the time scale for $R \gg 1$ ) was fixed, while $C_{12}$ (the intrabath dipolar coupling) was varied to obtain different values of $R=\Delta A / C_{12}$.

By means of a detailed theoretical analysis, we can show that average Hamiltonian theory is valid if (i) $\left|\mathbf{h}_{u}+\mathbf{h}_{d}\right| \gg$
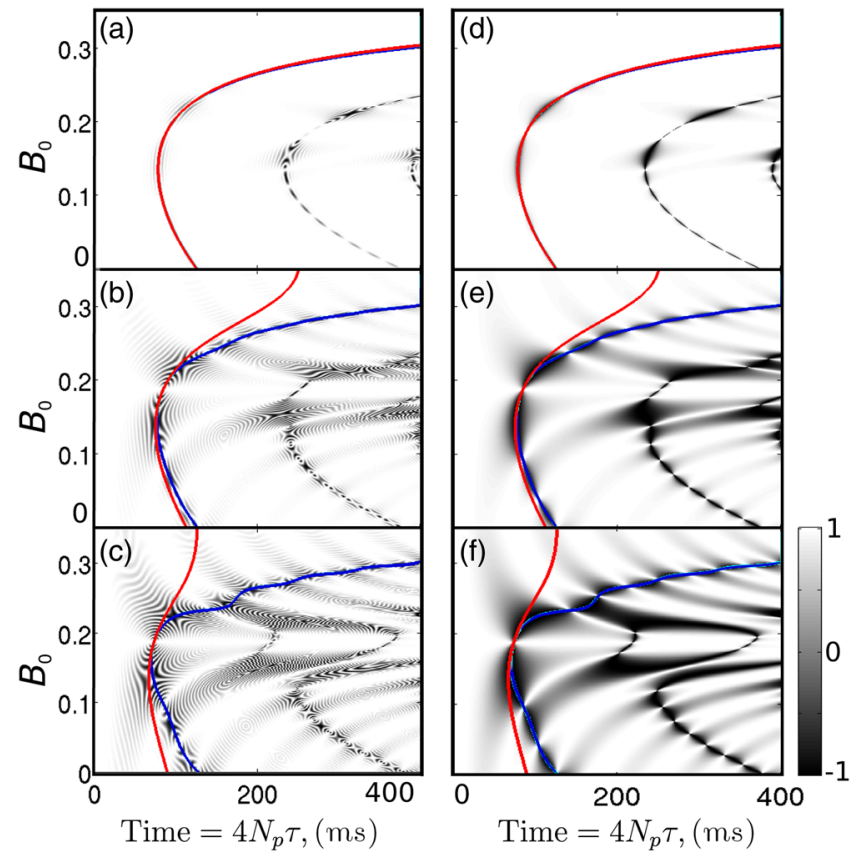

FIG. 4. Comparisons between the dip positions obtained with Eq. (4) (blue line) and average Hamiltonian theory Eq. (7) (red line) for the full coherence function (left) as well as its envelope (right). Within the field sweep there are global weak-coupling points (e.g., $B_{0} \approx 0.19 \mathrm{~T}$ ) where there is weak coupling regardless of the cluster properties and where the decoherence envelope collapses into a single sharp dip, a useful feature if high resolution is required: here, there is always good agreement with average Hamiltonian theory. These points correspond to so-called optimal working points $[31,32]$ of silicon donors. Hence, the advantage of such systems as future spin sensors, in addition to their very long $\sim 1$ s coherence times, is that a magnetic field sweep could tune the dynamics from the weak- to strong-coupling regimes. (a),(d) $R=100$; (b),(e) $R=20$; (c),(f) $R=10$. $\left|\mathbf{h}_{u}-\mathbf{h}_{d}\right|$ or if (ii) $\left|\mathbf{h}_{u}-\mathbf{h}_{d}\right| \gg\left|\mathbf{h}_{u}+\mathbf{h}_{d}\right|$. Condition (i) corresponds to the weak-coupling regimes typical of NV sensing experiments, where $\omega_{u} \simeq \omega_{d}$ and $\theta_{u} \simeq \theta_{u}$; it is also the regime of the optimal working points, where average Hamiltonian theory is always valid. Regime (ii) is not typical of sensing experiments: for the spin- $1 / 2$ system of Fig. 4, it would correspond to the spins nearly antialigned; thus, $P_{u} \simeq-P_{d}$. For Fig. 4, condition (ii) implies $(\Delta A)^{2}\left(P_{u}-P_{d}\right)^{2} \gg\left(C_{12}\right)^{2}+(\Delta A)^{2}\left(P_{u}+P_{d}\right)^{2}$. In particular, for $P_{u} \approx-P_{d}$, we obtain the condition $\left|\Delta A\left(P_{u}-P_{d}\right)\right| \gg$ $\left|C_{12}\right|$. Noting that $\left|P_{u}-P_{d}\right| \simeq 0.2-2$, this means that average Hamiltonian theory is valid at all fields for large $R \gtrsim 100$, as shown in Fig. 4(a). However, for increasing intrabath dipolar coupling $C_{12}$, the theory ceases to be valid away from the small OWP regime in the center, as shown in Figs. 4(b) and 4(c) for smaller $R$.

\section{B. Detection of multispin clusters}

In this section, we apply the Floquet approach to the system depicted in Fig. 1(d): we compare the decoherence "fingerprint" of three independent spin pairs (analogous, formally, to the detection of three independent spins by NMR) with a 3-cluster, which in the absence of many-body interactions would give a similar signature.

For the 3-cluster, we take three spins, with hyperfine couplings $A_{k} \equiv A_{1}, A_{2}, A_{3}$ to the sensor spin and with mutual dipolar interactions $C_{i j} \equiv C_{12}, C_{23}, C_{31}$. Disregarding interactions, the energy cost of the spin flips is $\Delta_{i j}=A_{i}-A_{j}$.

For the independent pairs, we take three spin pairs, with the same dipolar interactions $C_{i j}$ as the 3-cluster, but which are independent of each other. To have similar frequencies as the 3-cluster, we must have similar energy cost of all three spin flips, and they must obey the cyclic condition of the 3-cluster $\Delta_{12}+\Delta_{23}+\Delta_{31}=0$. Pair 1 has two spins with interaction $C_{12}$ and a pair of hyperfine couplings $\left(A_{1}, A_{2}\right)$; pair 2 has interaction $C_{23}$ and hyperfine couplings $\left(A_{2}, A_{3}\right)$; pair 3 has $C_{31}$ and hyperfine couplings $\left(A_{3}, A_{1}\right)$. We take $C_{12}=C_{23}=(1.05 / 2 \pi) \mathrm{kHz}$ and $C_{31}=(2.2 / 2 \pi) \mathrm{kHz}$, realistic values for nuclear impurities in the silicon lattice. We take $A_{1}=(180 / 2 \pi) \mathrm{kHz}, A_{3}=(100 / 2 \pi) \mathrm{kHz}$, and $A_{2}=0$; thus, our pairs correspond to $R \simeq 100-40$ (as defined in Fig. 3), so the interactions are sufficiently weak to make their detection challenging but sufficiently strong to, below, illustrate important features. The choice of $A_{2}=0$ does not involve much loss of generality. If a state-dependent Hamiltonian is chosen, the $A_{1}, A_{2}, A_{3}$ values can be shifted by an arbitrary constant without perturbing the dynamics. If the full Hamiltonian is considered, there can be higherorder effects such as hyperfine mediated corrections to $C_{i j}$. This correction is very small for our parameters but is tested by full numerics below.

Solution of total Hamiltonian.-First, we set aside all pseudospin approximations and do diagonalizations of the full Hamiltonian followed by time propagation, using 


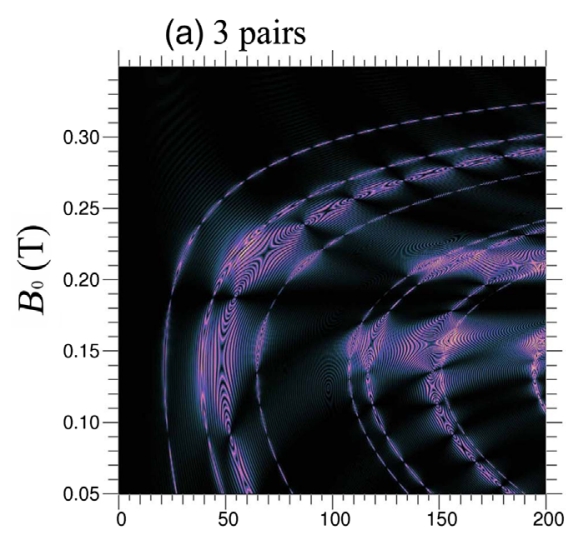

(b) 3-cluster

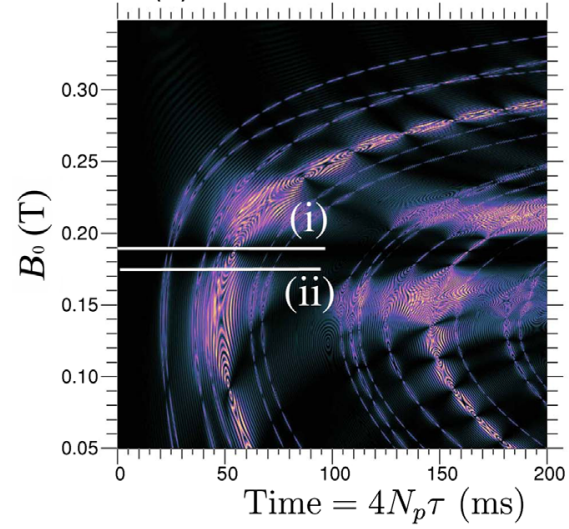

(i)

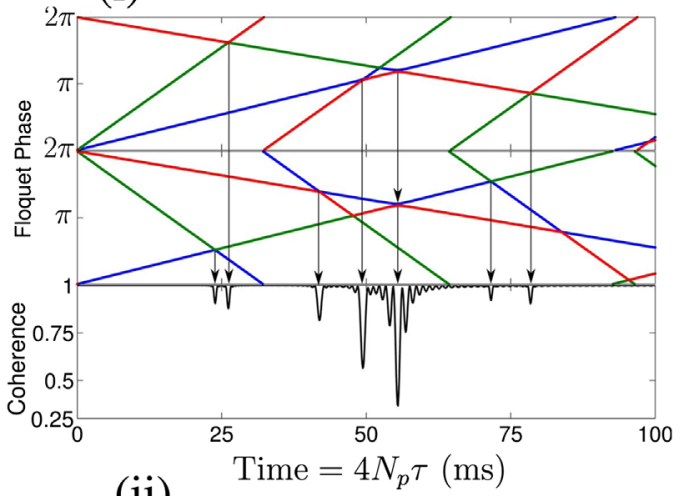

(ii)

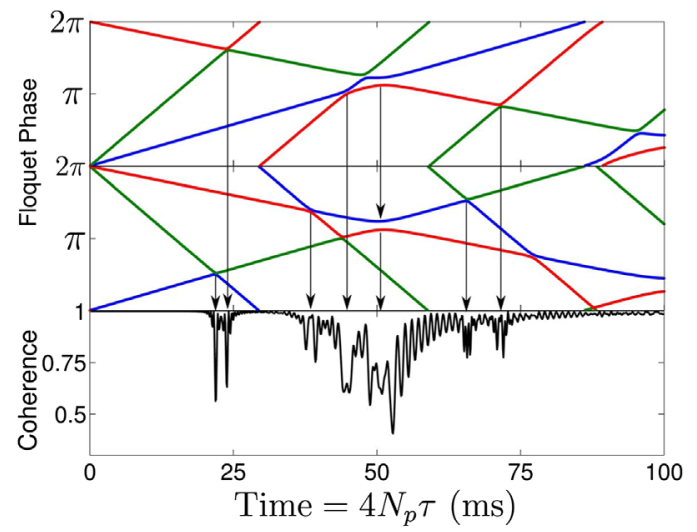

FIG. 5. Fingerprinting multiple environmental spin cluster pairs via their decoherence "bar codes" illustrates the effect of 3-body correlations. The figure shows the coherence as a function of magnetic field $B_{0}$ and pulse interval $\tau$, calculated with a full numerical propagation under the total Hamiltonian for $N_{p}=100$. Panel (a) denotes three independent pairs while panel (b) shows three interacting spins, with otherwise equivalent dipolar couplings and intrabath interactions as illustrated in Fig. 1(b). One evident difference (and signature of a cluster of three spins) is the doublets due to the two separate subspaces of the three interacting spins. The splittings are directly related to the interactions. For the 3-cluster, in fact, there is a secular contribution from interactions between spins, greatly amplifying their contribution. The two right-hand panels (i) and (ii) show single traces corresponding to the cuts in (b) as well as the six corresponding eigenphases: in case (i), in a weak-coupling regime the dips are narrow and the eigenphases behave like three independent pairs; the eigenvalues correspond to conjugate pairs (with blue, red, and green lines denoting the three pairs). In case (ii) there is stronger coupling, the avoided crossings of the corresponding eigenphases are broader, giving rise to the features shown in the coherence maps.

the complete 8 -state basis of the 3 -cluster as well as the complete basis of the bismuth sensor including the host nuclear spin. Thus, unlike Fig. 3, we do not use the analytical form for the parameter $P_{i}$; it emerges from the numerics. We evaluate the decoherence numerically rather than using Eq. (4). A similar calculation is carried out with the three disjoint pairs, then the decoherence was averaged over the thermal ensemble of nuclear spin states (of which there are eight for the 3 -cluster). Figure 5 shows maps of the coherence in the $\left(\tau, B_{0}\right)$ space in both cases.

One conclusion to be drawn from comparisons between full numerics and the analytical (one-pair) Eq. (4) is that the structure in Fig. 3 is surprisingly robust; without bath state averaging, full numerics give similar structure to Fig. 3 [obtained from Eq. (4) for one bath state].

One striking feature of the 3-cluster decoherence map in Fig. 5(b) is that some lines are split into "doublets" with very similar structure. The origin of these is in the average over the bath states; examining maps for the individual 8 bath states, we see that while the $I_{z}= \pm 3 / 2$ cluster states $|\uparrow \uparrow \uparrow\rangle$ and $|\downarrow \downarrow \downarrow\rangle$ make no appreciable contribution, the doublets arise from the separate $I^{z}= \pm 1 / 2$ subspaces, which do not mix. In other words, the $|\uparrow \uparrow \downarrow\rangle,|\uparrow \downarrow \uparrow\rangle$, and $|\downarrow \uparrow \uparrow\rangle$ states with total quantum number $I^{z}=+1 / 2$ interact only weakly with the equivalent $I^{z}=-1 / 2$ subspace, but each provides a locus of dips with a slightly different shift. In contrast to the spin pairs, in the case of the 3 -cluster, the secular Ising $\left(C_{j k} \hat{I}_{z j} \hat{I}_{z k}\right)$ components yield a nontrivial dynamical effect.

Figures 5(i) and 5(ii) also show a cut for two field values and compare with the behavior of the Floquet eigenstates. We can see that near the "weak-coupling" regime of optimal working points (i), the dips are sharp and narrow as are the avoided crossings. In contrast, away from the OWP point, avoided crossings are broader and even overlap 


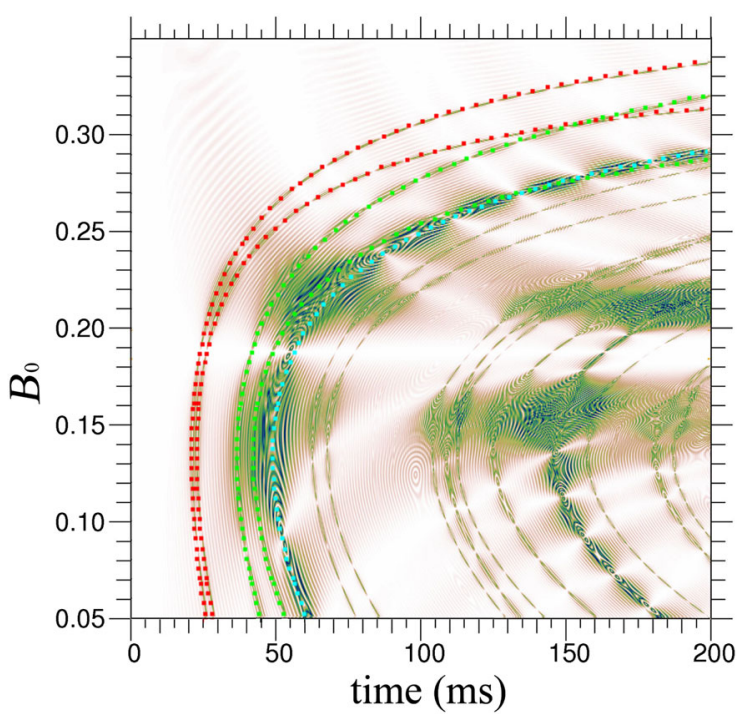

FIG. 6. Decoherence for an interacting cluster of three spins (3-cluster). The colored lines show comparisons with Eq. (10), showing excellent agreement with numerics obtained by diagonalization of the full joint sensor-cluster Hamiltonian.

(ii); the level splitting is much larger. We can estimate the point where two eigenvalues will collide and, hence, $\tau_{\text {dip }}$ by exploiting the fact that in either case, the average Hamiltonian theory value is not too far from the accurate value $\bar{\tau}_{\text {dip }} \simeq \tau_{\text {dip }}$. We estimate Floquet quasienergies by considering only the diagonals. We obtain

$$
\epsilon_{l}=\frac{1}{2}\left(A_{i}-A_{j}-A_{k}\right)\left(P_{u}+P_{d}\right)+C_{i j}+C_{i k}-C_{j k},
$$

where $i, j, k \equiv 1,2,3$ or cyclic permutations give $\epsilon_{l=1,2,3}$ quasienergies.

Thus, we estimate the dip positions from the fact that the quasienergies represent the gradients of the spectral lines in Figs. 5(i) and 5(ii); hence, we estimate the degeneracy point,

$$
\tau_{\mathrm{dip}}^{(l m)} \simeq \frac{2 \pi}{\epsilon_{l}-\epsilon_{m}}
$$

for the dip arising from the difference between the $l$ th and $m$ th quasienergy. One finding is that the secular contribution from the dipolar coupling greatly amplifies the effect of the (usually weaker) $C_{i j}$ dipolar coupling between the nuclei, as it is a linear contribution. This is in contrast to disjoint pairs; if the dipolar coupling is weak, since $\omega_{i}= \pm \frac{1}{4} \sqrt{C_{12}^{2}+\left(P_{i} \Delta A\right)^{2}}$, for $C_{12} \ll P_{i} \Delta A$ the nonsecular contributions in the disjoint pairs represent a very small quadratic shift.

In terms of the interaction strengths, the two dips of the first doublet correspond to

$$
\tau_{\mathrm{dip}}^{( \pm 12)} \simeq \frac{2 \pi}{\left|\Delta_{12}\left(P_{u}+P_{d}\right) \pm 2\left(C_{31}-C_{23}\right)\right|},
$$

and similarly for other doublets.

In Fig. 6, we compare values from Eq. (10) with the full numerics. Thus, the mean position exposes the value of $\Delta_{12}$, while the splittings expose the dipolar coefficients.

\section{CONCLUSIONS}

The extension of technologies such as MRI and NMR to the nanoscale is an outstanding technical challenge that is leading not only rapid experimental progress but also the development of new methods to analyze data and to optimize information gathering on the atomic-scale structure.

Motivated by this, in the present work we introduce Floquet spectroscopy as an insightful new paradigm for understanding and analysis of spin-sensing experiments. The approach is universally valid for any type of periodic driving, whether resonant or not. Hence, here potential applications are explored for analysis of different physical regimes and sensors that are not necessarily associated with a single sharp resonant "dip," but may nevertheless potentially still offer well-delineated features.

Our key findings are as follows. (i) There is an underlying structure associated with Floquet avoided crossings and the Floquet spectrum, which is potentially information rich. It represents an envelope on the usually studied coherence dips with a shape controlled by the widths of the avoided crossings. (ii) The Floquet approach also clarifies regimes where the commonly used average Hamiltonian theory methods will fail. (iii) The method's generality extends beyond single-spin and pseudospin systems and is also useful for higher-dimensional spin systems, and potentially any dynamical-decoupling protocol, provided it is temporally periodic.

\section{ACKNOWLEDGMENTS}

We are very grateful to Setrak Balian, Gary Wolfowicz, and Gavin Morley for helpful discussions. J. E. L. acknowledges a studentship from the Engineering and Physical Sciences Research Council (EPSRC). R. B. L. was supported by Hong Kong RGC/CRF CUHK4/CRF/12G.

\section{APPENDIX A: FLOQUET SPECTRUM}

\section{Coherence minima and avoided crossings}

A key result of the present work is that the coherence dips associated with single-spin sensing are associated with avoided crossings of the underlying Floquet spectrum, and in this appendix, this conjecture is justified.

In our study, we consider the important class of spinsensing experiments for which an electronic sensor spin $S$ is coupled to each environmental nucleus via the effective Hamiltonian: 


$$
\hat{H} \approx\left\langle i\left|\hat{S}_{z}\right| i\right\rangle \mathbf{A} \cdot \mathbf{I},
$$

where $i=u, d$ and $\mathbf{A}$ is a vector representing the hyperfine interaction. The dependence on $S_{z}$ arises only because of the large energy difference between electronic and nuclear states; in the case of NV centers, the above is valid only for magnetic fields of magnitude and orientation which do not mix the electronic states. The result is a state conditional Hamiltonian,

$$
\hat{H}=\frac{1}{\sqrt{2}}\left[|u\rangle\left\langle u\left|\otimes H_{u}+\right| d\right\rangle\langle d| \otimes H_{d}\right],
$$

where the $H_{i}$ are the effective bath Hamiltonians discussed in Sec. II A, so that an initial joint sensor-target spin state $\psi(0) \simeq(1 / \sqrt{2})(|u\rangle+|d\rangle) \mathcal{B}(0)$ evolves into an (in general) entangled state,

$$
\psi(t)=\frac{1}{\sqrt{2}}\left[|u\rangle \mathcal{B}_{u}(t)+|d\rangle \mathcal{B}_{d}(t)\right] .
$$

Experiments probe the coherence $\mathcal{L}(t)=\left\langle S^{+}\right\rangle$. While experimental comparison involves averaging over thermal bath states $\operatorname{Tr}\left[\rho S^{+}\right]$, without loss of generality we consider a pure state $\mathcal{L}=\left\langle\psi(t)\left|S^{+}\right| \psi(t)\right\rangle \propto\left\langle\mathcal{B}_{u}(t) \mid \mathcal{B}_{d}(t)\right\rangle$.

Maximum entanglement occurs whenever $\left\langle\mathcal{B}_{u}(t) \mid \mathcal{B}_{d}(t)\right\rangle=$ 0 . However, the general condition for a minimum or dip to be observed is in fact $\left|\mathcal{B}_{u}(t)\right\rangle=-\left|\mathcal{B}_{d}(t)\right\rangle$. This is regardless of the particular dynamical decoupling sequence applied. The key question for design of an experimental pulse sequence (say, CPMG) is which pulse interval $\tau$ and total pulse number $N \equiv 2 N_{p}$ will correspond to underlying quantum evolution,

$$
\left\langle\mathcal{B}_{u}\left(t=4 N_{p} \tau\right) \mid \mathcal{B}_{d}\left(t=4 N_{p} \tau\right)\right\rangle=-1,
$$

and thus a minimum in the function $\mathcal{L}$, which to within an unimportant normalization factor we take $\mathcal{L}=$ $\left\langle\mathcal{B}_{u}(t) \mid \mathcal{B}_{d}(t)\right\rangle$ (we note that for decoherence experiments probing $|\mathcal{L}|$, this in fact corresponds to a maximum in the coherence).

The Floquet approach is based on the premise that for any periodically driven quantum system, the Floquet states $\Phi_{j}$ fulfil the same role as eigenstates of a Hamiltonian in a time-independent system. Thus, if the initial quantum state is projected into a Floquet basis, i.e., $\mathcal{B}(0)=\sum_{l} a_{l} \Phi_{l}$, then its temporal evolution is known for all time.

A central finding for the present work is that for the pulsed dynamical decoupling, the eigenspectrum is independent of the sensor spin state; thus, $\mathcal{E}_{l}^{(u)}=\mathcal{E}_{l}^{(d)} \equiv \mathcal{E}_{l}(\tau)$, where the Floquet eigenspectrum $\mathcal{E}_{l}(\tau)$ is a function of the experimentally chosen pulse interval $\tau$.

The above results are proved in the next section, but we use them now to explain why coherence minima are associated with avoided crossings. Since the Floquet spectra are the same, if there is an avoided crossing and thus a near degeneracy, $e^{\mathcal{E}_{l}(\tau)} \simeq e^{\mathcal{E}_{k}(\tau)}$, in the $u$ subspace of sensor states, there will simultaneously be an avoided crossing in the lower $d$ subspace of sensor states.

\section{Avoided crossings for two-level system}

Although the eigenspectra are the same, in general the corresponding eigenstates or Floquet states are not. $\Phi_{d}(\tau) \neq$ $\Phi_{u}(\tau)$ for arbitrary $\tau$. Hence, the temporal evolution,

$\mathcal{B}_{u}(t)=a_{u+} \Phi_{u+} e^{-i N_{p} \mathcal{E}(\tau)}+a_{u-} \Phi_{u-} e^{+i N_{p} \mathcal{E}(\tau)} \neq$
$\mathcal{B}_{d}(t)=a_{d+} \Phi_{d+} e^{-i N_{p} \mathcal{E}(\tau)}+a_{d_{-}} \Phi_{d_{-}} e^{+i N_{p} \mathcal{E}(\tau)}$,

and, thus, entanglement with the sensor is established since the sensor-target $\mathcal{B}_{u, d}(t)$ state is no longer factorizable.

One exception occurs for $\tau=0$, where all the Floquet states reduce to the unperturbed (thermal states). For a two-state system, without loss of generality, $\Phi_{u+}(\tau=0)=$ $\Phi_{d+}(\tau=0)=|\uparrow\rangle$, or alternatively, $\Phi_{u-}(\tau=0)=\Phi_{d_{-}}(\tau=$ $0)=|\downarrow\rangle$.

Another, most interesting, exception is at a level crossing, where the eigenstates take the same form. It is a textbook result for level crossings (also known as LandauZener transitions) that the unperturbed states are maximally mixed and become sums and differences of the unperturbed states. The implication for the present case is that the Floquet states for both upper and lower state must coincide at approximately $\frac{1}{\sqrt{2}}(|\downarrow\rangle \pm|\uparrow\rangle)$. This allows for two distinct possibilities.

(i) In the first case,

$$
\begin{array}{ll}
\Phi_{u+} & =\Phi_{d+} \simeq \frac{1}{\sqrt{2}}(|\downarrow\rangle+|\uparrow\rangle) \quad \text { and } \\
\Phi_{u-} & =\Phi_{d-} \simeq \frac{1}{\sqrt{2}}(|\downarrow\rangle-|\uparrow\rangle) .
\end{array}
$$

This possibility is the trivial case where the Floquet states for the upper and lower sensor state are identical. There is never any difference in the evolution, no entanglement, and so $\left\langle\mathcal{B}_{u}(t) \mid \mathcal{B}_{d}(t)\right\rangle=+1$.

(ii) In the second case,

$$
\begin{aligned}
& \Phi_{u+}=\Phi_{d-} \simeq \frac{1}{\sqrt{2}}(|\downarrow\rangle+|\uparrow\rangle) \quad \text { and } \\
& \Phi_{u-}=\Phi_{d+} \simeq \frac{1}{\sqrt{2}}(|\downarrow\rangle-|\uparrow\rangle) .
\end{aligned}
$$

In this case, $\left\langle\mathcal{B}_{u} \mid \mathcal{B}_{d}\right\rangle=\cos 2 N_{p} \mathcal{E}(\tau)$, which may, for an appropriate choice of $N_{p} \approx \pi / 2 \mathcal{E}$, attain the minimal value for a $\operatorname{dip}\left\langle\mathcal{B}_{u} \mid \mathcal{B}_{d}\right\rangle=-1$.

Diagonalization of the two-dimensional unitary matrix is straightforward (see Ref. [25]), and it is clear its eigenvalues must be conjugate pairs, $\lambda_{ \pm}=e^{ \pm i \mathcal{E}}$. For a two-level case, the avoided crossing condition is $\lambda_{+}=\lambda_{-}$, and, hence, coherence dips must occur at $\mathcal{E} \simeq 0, \pi, 2 \pi$ with case (i) occurring at $\mathcal{E} \simeq \pi$ and case (ii) occurring at $\mathcal{E} \simeq 0,2 \pi$. 


\section{Coherence minima for the general case}

For a spin cluster of arbitrary size, an initial pure state can be projected into the upper or lower Floquet basis:

$$
|\mathcal{B}(0)\rangle=\sum_{l}^{D}\left\langle\Phi_{i l} \mid \mathcal{B}(0)\right\rangle\left|\Phi_{i l}\right\rangle,
$$

where $\left|\Phi_{i l}\right\rangle$ are Floquet eigenstates for the upper $(i=u)$ and lower $(i=d)$ sensor state, respectively, and $D$ is the dimension of the one-period unitary evolution operator. The state after a time $t=4 N_{p} \tau$ is then

$$
\left|\mathcal{B}_{i}(t)\right\rangle=\sum_{l}^{D} e^{-i N_{p} \mathcal{E}_{l}}\left\langle\Phi_{i l} \mid \mathcal{B}(0)\right\rangle\left|\Phi_{i l}\right\rangle,
$$

where $\mathcal{E}_{l}$ are the eigenphases.

Thus, the coherence decay of the sensor spin $\left[\mathcal{L}(t)=\left\langle\mathcal{B}_{l}(t) \mid \mathcal{B}_{u}(t)\right\rangle\right]$ is given in a Floquet basis by

$$
\mathcal{L}\left(t=N_{p} 4 \tau\right)=\sum_{l, l^{\prime}}^{D} e^{-i N_{p}\left(\mathcal{E}_{l}-\mathcal{E}_{l^{\prime}}\right)}\left|a_{l}\right|^{2}\left|\left\langle\Phi_{d l^{\prime}} \mid \Phi_{u l}\right\rangle\right|^{2} .
$$

Here, $\left|a_{l}\right|^{2}=\left|\left\langle\Phi_{u l} \mid \mathcal{B}(0)\right\rangle\right|^{2}$.

The target spins are in fact in a thermal ensemble, which, given small nuclear energy scale, are all equally likely. Thus, we must average over the thermal bath states and calculate $\langle\mathcal{L}\rangle=(1 / D) \sum_{j}^{D} \mathcal{L}_{j}$, where $\mathcal{L}_{j}$ is the coherence function evaluated for the bath initially in the $j$ th thermal bath state, $\left|\mathcal{B}_{j}(0)\right\rangle$. Under the thermal average, we find $\sum_{j}\left\langle\Phi_{u l} \mid \mathcal{B}_{j}(0)\right\rangle\left\langle\mathcal{B}_{j}(0) \mid \Phi_{u l}\right\rangle=1$. This produces Eq. (3). While $\mathcal{L}$ for a pure state is complex, the (in any case small) imaginary part vanishes under the bath average and we consider only the real part of $\mathcal{L}$.

Returning briefly to the simplest case of $D=2$, treated in the previous section, Eq. (A10) may be rewritten as

$$
\langle\mathcal{L}(\tau)\rangle=1-2\left|\left\langle\Phi_{d-} \mid \Phi_{u+}\right\rangle\right|^{2} \sin ^{2}\left(N_{p} \frac{\mathcal{E}_{1}-\mathcal{E}_{2}}{2}\right),
$$

which is of the form $\mathcal{L}(\tau)=1-F(\tau) f\left(N_{p}, \tau\right)$, where $f\left(N_{p}, \tau\right) \in[0,1]$ is a pulse-number-dependent oscillation. If we disregard the oscillation, we obtain the pulse-numberindependent minimal bound of the coherence function given by $\mathcal{L}_{\text {env }}(\tau)=1-F(\tau)$, which we call the coherence envelope.

Even if these envelopes are not necessarily sharp "dips" (especially in strong-coupling regimes), they can correspond to sharply delineated structures (for both NV centers and donors), which should still be observable experimentally and can yield valuable information about the atomicscale structure. We show above that the dips occur when $\left|\Phi_{u+}\right\rangle=\left|\Phi_{d_{-}}\right\rangle$.

For a general bath, of arbitrary dimension $D$, we can rearrange Eq. (A10) using only orthonormality and completeness of the eigenstates $\sum_{k^{\prime}}\left|\left\langle\Phi_{d k^{\prime}} \mid \phi\right\rangle\right|^{2}=1$ into paired contributions:

$$
\begin{aligned}
\langle\mathcal{L}\rangle \equiv & 1-\sum_{l<l^{\prime}}\left[\left|\left\langle\Phi_{d l^{\prime}} \mid \Phi_{u l}\right\rangle\right|^{2}+\left|\left\langle\Phi_{d l} \mid \Phi_{u l^{\prime}}\right\rangle\right|^{2}\right] \\
& \times \sin ^{2}\left(N_{p} \frac{\mathcal{E}_{l}-\mathcal{E}_{l^{\prime}}}{2}\right) .
\end{aligned}
$$

This is, again, composed of pulse-number-independent envelopes superimposed with pulse-number-dependent oscillations. For a minimum, we require the term in square brackets to be maximized; this will occur at a level crossing between a given pair of eigenstates $l$ and $l^{\prime}$ as argued in the previous section. For $D>2$, level crossings between $\mathcal{E}_{l} \approx$ $\mathcal{E}_{l^{\prime}}$ occur at arbitrary $\mathcal{E}_{l}$ and no longer at $\mathcal{E}_{l, l^{\prime}} \approx \pi$. Dips occurring at the point for which $\left|\left\langle\Phi_{d l^{\prime}} \mid \Phi_{u l}\right\rangle\right|^{2}=1$ and $\left|\left\langle\Phi_{d l} \mid \Phi_{u l^{\prime}}\right\rangle\right|^{2}=1$ generalize, to arbitrary dimension, the two-state orthogonality condition that $\left|\Phi_{u+}\right\rangle=\left|\Phi_{d-}\right\rangle$.

\section{APPENDIX B: SYMMETRY OF EIGENPHASES FOR CPMG CONTROL}

For decoupling sequences like CPMG, the Floquet phases are independent of the sensor spin state, regardless of the dimensionality of the bath states; i.e., $\mathcal{E}_{l}^{(u)}=\mathcal{E}_{l}^{(d)} \equiv \mathcal{E}_{l}$. To show this, we first construct the basic propagator, for total period $\tau_{\text {tot }}=4 \tau$, which is to be repeated periodically:

$$
\begin{aligned}
\hat{T}_{(u)}^{(2)}(4 \tau) & =\hat{T}_{(u)}(\tau) \hat{T}_{(d)}(\tau) \hat{T}_{(d)}(\tau) \hat{T}_{(u)}(\tau) \\
& \equiv \hat{T}_{(u)}(2 \tau) \hat{T}_{(d)}(2 \tau), \\
\hat{T}_{(d)}^{(2)}(4 \tau) & =\hat{T}_{(d)}(\tau) \hat{T}_{(u)}(\tau) \hat{T}_{(u)}(\tau) \hat{T}_{(d)}(\tau) \\
& \equiv \hat{T}_{(d)}(2 \tau) \hat{T}_{(u)}(2 \tau) .
\end{aligned}
$$

We can then obtain the eigenvalues for $\hat{T}_{(u)}^{(2)}$ :

$$
\hat{T}_{(u)}^{(2)}(4 \tau)\left|\Phi_{u l}\right\rangle=\hat{T}_{(u)}(2 \tau) \hat{T}_{(d)}(2 \tau)\left|\Phi_{u l}\right\rangle=e^{-i \mathcal{E}_{l}}\left|\Phi_{u l}\right\rangle .
$$

Here, $\exp \left(-i \mathcal{E}_{l}\right)$ is the $l$ th eigenvalue of $\hat{T}_{(u)}^{(2)}$. If we apply the half-period operator, $\hat{T}_{(d)}(2 \tau)$, we get

$$
\hat{T}_{(d)}(2 \tau) \hat{T}_{(u)}(2 \tau) \hat{T}_{(d)}(2 \tau)\left|\Phi_{u l}\right\rangle=e^{-i \mathcal{E}_{l}} \hat{T}_{(d)}(2 \tau)\left|\Phi_{u l}\right\rangle ;
$$

this is equivalent to

$$
\hat{T}_{(d)}^{(2)}(4 \tau) \hat{T}_{(d)}(2 \tau)\left|\Phi_{u l}\right\rangle=e^{-i \mathcal{E}_{l}} \hat{T}_{(d)}(2 \tau)\left|\Phi_{u l}\right\rangle,
$$

Thus, $\exp \left(-i \mathcal{E}_{l}\right)$ is also an eigenvalue of $\hat{T}_{(d)}^{(2)}$. Equation (B4) implies that $\hat{T}_{(d)}(2 \tau)\left|\Phi_{u l}\right\rangle$ is an eigenstate of $\hat{T}_{(d)}^{(2)}$, i.e., $\hat{T}_{(d)}(2 \tau)\left|\Phi_{u l}\right\rangle \propto\left|\Phi_{d l}\right\rangle$, where the factor of proportionality is a complex phase $\exp \left(i \mu_{l d}\right)$. Similarly, 


$$
\hat{T}_{(u)}(2 \tau)\left|\Phi_{d l}\right\rangle=\exp \left(i \mu_{l u}\right)\left|\Phi_{u l}\right\rangle
$$

for which $\mu_{l d}+\mu_{l u}=\mathcal{E}_{l}$. From this we see that each Floquet state $\left|\Phi_{u l}\right\rangle,\left|\Phi_{d l}\right\rangle$ is the half-period evolution of the other (up to a complex phase).

\section{APPENDIX C: PULSES OF FINITE DURATION}

Provided that $\hat{T}_{(u, d)}^{(2)}(4 \tau)$ can be decomposed into products of subpropagators, as in Eq. (C2), the $\pi$ pulses do not have to be of very short duration. If we write

$$
\begin{aligned}
& \hat{T}_{(u)}(2 \tau+2 \delta)=\hat{T}_{(u)}(\tau) T_{\pi}(2 \delta) \hat{T}_{(d)}(\tau), \\
& \hat{T}_{(d)}(2 \tau+2 \delta)=\hat{T}_{(d)}(\tau) T_{\pi}(2 \delta) \hat{T}_{(u)}(\tau),
\end{aligned}
$$

then we can write the full propagator in the same form as previously:

$$
\begin{aligned}
& \hat{T}_{(u)}^{(2)}\left(4 \tau^{\prime}\right)=\hat{T}_{(u)}\left(2 \tau^{\prime}\right) \hat{T}_{(d)}\left(2 \tau^{\prime}\right), \\
& \hat{T}_{(d)}^{(2)}\left(4 \tau^{\prime}\right)=\hat{T}_{(d)}\left(2 \tau^{\prime}\right) \hat{T}_{(u)}\left(2 \tau^{\prime}\right),
\end{aligned}
$$

but with $\tau^{\prime}=\tau+\delta$. Then all the above follow: the pulses of finite duration are still assumed to be $\pi$ pulses, but there can be some arbitrary evolution of the system during the finite interval $\delta$, but properties such as the state independence of the Floquet phases still hold.

[1] F. Jelezko, T. Gaebel, I. Popa, M. Domhan, A. Gruber, and J. Wrachtrup, Observation of Coherent Oscillation of a Single Nuclear Spin and Realization of a Two-Qubit Conditional Quantum Gate, Phys. Rev. Lett. 93, 130501 (2004).

[2] L. Childress, M. V. Gurudev Dutt, J. M. Taylor, A. S. Zibrov, F. Jelezko, J. Wrachtrup, P. R. Hemmer, and M. D. Lukin, Coherent Dynamics of Coupled Electron and Nuclear Spin Qubits in Diamond, Science 314, 281 (2006).

[3] J. R. Maze, P. L. Stanwix, J. S. Hodges, S. Hong, J. M. Taylor, P. Cappellaro, L. Jiang, M. V. Gurudev Dutt, E. Togan, A. S. Zibrov, A. Yacoby, R. L. Walsworth, and M. D. Lukin, Nanoscale Magnetic Sensing with an Individual Electronic Spin in Diamond, Nature (London) 455, 644 (2008).

[4] G. Balasubramanian, I. Y. Chan, R. Kolesov, M. Al-Hmoud, J. Tisler, C. Shin, C. Kim, A. Wojcik, P. R. Hemmer, A. Krueger, T. Hanke, A. Leitenstorfer, R. Bratschitsch, F. Jelezko, and J. Wrachtrup, Nanoscale Imaging Magnetometry with Diamond Spins Under Ambient Conditions, Nature (London) 455, 648 (2008).

[5] T. Muller, C. Hepp, B. Pingault, E. Neu, S. Gsell, M. Schreck, H. Sternschulte, D. Steinmller-Nethl, C. Becher, and M. Atature, Optical Signatures of Silicon-Vacancy Spins in Diamond, Nat. Commun. 5, 3328 (2014).

[6] J. Hansom, C. H. H. Schulte, C. Le Gall, C. Matthiesen, E. Clarke, M. Hugues, J. M. Taylor, and M. Atature,
Environment-Assisted Quantum Control of a Solid-State Spin via Coherent Dark States, Nat. Phys. 10, 725 (2014).

[7] P. Cappellaro, L. Jiang, J. S. Hodges, and M. D. Lukin, Coherence and Control of Quantum Registers Based on Electronic Spin in a Nuclear Spin Bath, Phys. Rev. Lett. 102, 210502 (2009).

[8] P. Neumann, R. Kolesov, B. Naydenov, J. Beck, F. Rempp, M. Steiner, V. Jacques, G. Balasubramanian, M. L. Markham, D. J. Twitchen, S. Pezzagna, J. Meijer, J. Twamley, F. Jelezko, and J. Wrachtrup, Quantum Register Based on Coupled Electron Spins in a Room-Temperature Solid, Nat. Phys. 6, 249 (2010).

[9] N. Bar-Gill, L. M. Pham, A. Jarmola, D. Budker, and R. L. Walsworth, Solid-State Electronic Spin Coherence Time Approaching One Second, Nat. Commun. 4, 1743 (2013).

[10] T. H. Taminiau, J. Cramer, T. van der Sar, V. V. Dobrovitski, and R. Hanson, Universal Control and Error Correction in Multi-Qubit Spin Registers in Diamond, Nat. Nanotechnol. 9, 171 (2014).

[11] H. Bernien, B. Hensen, W. Pfaff, G. Koolstra, M. S. Blok, L. Robledo, T. H. Taminiau, M. Markham, D. J. Twitchen, L. Childress, and R. Hanson, Heralded Entanglement between Solid-State Qubits Separated by Three Metres, Nature (London) 497, 86 (2013).

[12] J. Cai, F. Jelezko, M. B. Plenio, and A. Retzker, DiamondBased Single-Molecule Magnetic Resonance Spectroscopy, New J. Phys. 15, 013020 (2013).

[13] C. Mller, X. Kong, J.-M. Cai, K. Melentijevi, A. Stacey, M. Markham, D. Twitchen, J. Isoya, S. Pezzagna, J. Meijer, J. F. Du, M. B. Plenio, B. Naydenov, L. P. McGuinness, and F. Jelezko, Nuclear Magnetic Resonance Spectroscopy with Single Spin Sensitivity, Nat. Commun. 5, 4703 (2014).

[14] N. Zhao, J. L. Hu, S. W. Ho, J. T. K. Wan, and R. B. Liu, Atomic-Scale Magnetometry of Distant Nuclear Spin Clusters via Nitrogen-Vacancy Spin in Diamond, Nat. Nanotechnol. 6, 242 (2011).

[15] N. Zhao, J. Honert, B. Schmid, M. Klas, J. Isoya, M. Markham, D. Twitchen, F. Jelezko, R.-B. Liu, H. Fedder, and J. Wrachtrup, Sensing Single Remote Nuclear Spins, Nat. Nanotechnol. 7, 657 (2012).

[16] F. Shi, X. Kong, P. Wang, F. Kong, N. Zhao, R.-B. Liu, and J. Du, Sensing and Atomic-Scale Structure Analysis of Single Nuclear-Spin Clusters in Diamond, Nat. Phys. 10, 21 (2014).

[17] M. Loretz, J. M. Boss, T. Rosskopf, H. J. Mamin, D. Rugar, and C. L. Degen, Spurious Harmonic Response of Multipulse Quantum Sensing Sequences, Phys. Rev. X 5, 021009 (2015).

[18] R. Schirhagl, K. Chang, M. Loretz, and C. L. Degen, NitrogenVacancy Centers in Diamond: Nanoscale Sensors for Physics and Biology, Annu. Rev. Phys. Chem. 65, 83 (2014).

[19] J. H. Shirley, Solution of the Schrödinger Equation with a Hamiltonian Periodic in Time, Phys. Rev. 138, B979 (1965).

[20] A. Schmidt and S. Vega, The Floquet Theory of Nuclear Magnetic Resonance Spectroscopy of Single Spins and Dipolar Coupled Spin Pairs in Rotating Solids, J. Chem. Phys. 96, 2655 (1992); T. O. Levante, M. Baldus, B. H. Meier, and R. R. Ernst, Formalized Quantum Mechanical Floquet Theory and its Application to Sample Spinning in 
Nuclear Magnetic Resonance, Mol. Phys. 86, 1195 (1995); A. Schweiger and G. Jeschke, Principles of Pulse Electron Paramagnetic Resonance (Oxford University Press, Oxford, England, 2001).

[21] S. Kolkowitz, Q. P. Unterreithmeier, S. D. Bennett, and M. D. Lukin, Sensing Distant Nuclear Spins with a Single Electron Spin, Phys. Rev. Lett. 109, 137601 (2012).

[22] T. H. Taminiau, J. J. T. Wagenaar, T. van der Sar, F. Jelezko, V. V. Dobrovitski, and R. Hanson, Detection and Control of Individual Nuclear Spins Using a Weakly Coupled Electron Spin, Phys. Rev. Lett. 109, 137602 (2012).

[23] N. Zhao, S. W. Ho, and R. B. Liu, Decoherence and Dynamical Decoupling Control of Nitrogen Vacancy Center Electron Spins in Nuclear Spin Baths, Phys. Rev. B 85, 115303 (2012).

[24] A. Ajoy, U. Bissbort, M. D. Lukin, R. L. Walsworth, and P. Cappellaro, Atomic-Scale Nuclear Spin Imaging Using Quantum-Assisted Sensors in Diamond, Phys. Rev. X 5, 011001 (2015).

[25] See Supplemental Material at http://link.aps.org/ supplemental/10.1103/PhysRevX.5.041016 for further details of spin Hamiltonians and geometric methods.

[26] A. A. Morello, J. J. Pla, F. A. Zwanenburg, K. W. Chan, K. Y. Tan, H. Huebl, M. Möttönen, C. D. Nugroho, C. Yang, J. A. van Donkelaar, A. D. C. Alves, D. N. Jamieson, C. C. Escott, L. C. L. Hollenberg, R. G. Clark, and A. S. Dzurak, Single-Shot Readout of an Electron Spin in Silicon, Nature (London) 467, 687 (2010).

[27] J. J. Pla, K. Y. Tan, J. P. Dehollain, W. H. Lim, J. J. L. Morton, D. N. Jamieson, A. S. Dzurak, and A. Morello,
High-Fidelity Readout and Control of a Nuclear Spin Qubit in Silicon, Nature (London) 496, 334 (2013).

[28] J. T. Muhonen, J. P. Dehollain, A. Laucht, F. E. Hudson, T. Sekiguchi, K. M. Itoh, D. N. Jamieson, J. C. McCallum, A.S. Dzurak, and A. Morello, Storing Quantum Information for 30 Seconds in a Nanoelectronic Device, Nat. Nanotechnol. 9, 986 (2014).

[29] J. J. Pla, F. A. Mohiyaddin, K. Y. Tan, J. P. Dehollain, R. Rahman, G. Klimeck, D. N. Jamieson, A. S. Dzurak, and A. Morello, Coherent Control of a Single ${ }^{29} \mathrm{Si}$ Nuclear Spin Qubit, Phys. Rev. Lett. 113, 246801 (2014).

[30] R.-B. Liu, W. Yao, and L. J. Sham, Control of Electron Spin Decoherence Caused by Electron-Nuclear Spin Dynamics in a Quantum Dot, New J. Phys. 9, 226 (2007); W. Yao, R.-B. Liu, and L. J. Sham, Restoring Coherence Lost to a Slow Interacting Mesoscopic Spin Bath, Phys. Rev. Lett. 98, 077602 (2007).

[31] S. J. Balian, G. Wolfowicz, J. J. L. Morton, and T. S. Monteiro, Quantum-Bath-Driven Decoherence of Mixed Spin Systems, Phys. Rev. B 89, 045403 (2014).

[32] M. H. Mohammady, G. W. Morley, and T. S. Monteiro, Bismuth Qubits in Silicon: The Role of EPR Cancellation Resonances, Phys. Rev. Lett. 105, 067602 (2010).

[33] G. W. Morley, P. Lueders, M. H. Mohammady, S. J. Balian, G. Aeppli, C. W. M. Kay, W. M. Witzel, G. Jeschke, and T. S. Monteiro, Quantum Control of Hybrid Nuclearelectronic Qubits, Nat. Mater. 12, 103 (2013).

[34] G. Wolfowicz, A. M. Tyryshkin, R. E. George, H. Riemann, N. V. Abrosimov, P. Becker, H.-J. Pohl, M. L. W. Thewalt, S. A. Lyon, and J. J. L. Morton, Atomic Clock Transitions in Silicon-Based Spin Qubits, Nat. Nanotechnol. 8, 561 (2013). 